\title{
Assessment of Competitive Edge of Major Global Semiconductor Vendors for Self-Driving Solutions (Level 3 and Above)- Evaluation of Qualcomm, Intel, and Nvidia
}

\author{
Jae-Kyung Kim,1) Jon-Mo Yoon²), Bong-Soo Lee3)
}

\begin{abstract}
One of the important issues in the era of the Fourth Industrial Revolution is the commercialization of self-driving technology. Also, core semiconductor technology and support capabilities are very important to continuously develop self-driving technology. Along with looking at the status of the global semiconductor market, this paper's ultimate goal is to analyze the competitive edge of global innovative semiconductor companies' self-driving solutions by dividing them into technology innovation, knowledge management, human resources, sustainable management, and commercial business factors. This paper finally provides milestones and counterplans of the Korean government, local car OEMs, and domestic semiconductor providers on future self-driving solutions. For this paper's design and methodology, we extracted major factors for a competitive edge study using feedback and compelling data provided by experts and professors who have engaged in self-driving solutions, as well as the automobile industry. Based on the finding of the study, the researchers found the comparative competitive edge level of global major semiconductor makers for each factor. This paper would also help the Korean government develop a strategy to cope with the self-driving technology trend and global semiconductor market expansion.
\end{abstract}

Keywords: Competitive Edge, Self-Driving, Semiconductor, Intel, Nvidia, Qualcomm

\section{Introduction}

With the recent advent of the Fourth Industrial Revolution, a variety of products and convergence technologies have emerged, focusing on the latest technologies such as big data, artificial intelligence, robots, and self-driving cars. In addition, the products and technologies

Received(May 12, 2020), Review Result(1st: June 29, 2020, 2nd: August 18, 2020), Accepted(September 25, 2020)

1) (VP of Qualcomm, Author) 06060 17th FL. POBA Gangnam Tower, 343, Hakdong-ro, Gangnam-gu, Seoul, Korea

email: jkkim0521@hanmail.net

2) (Professor, Co-Author) 16890 Dept. Liberal Arts, DanKook Univ., Jukjeon-ro, Suji-gu, Yongin-si, Gyeonggi-do, Korea

email: jyoon2010@naver.com

3) (Professor, Corresponding Author) 16890 Dept. International Trade, DanKook Univ., Jukjeon-ro, Suji-gu, Yongin-si, Gyeonggi-do, Korea

email: leebongsoo@dankook.ac.kr 
Assessment of Competitive Edge of Major Global Semiconductor Vendors for Self-Driving Solutions (Level 3 and Above)- Evaluation of Qualcomm, Intel, and Nvidia

needed at home and in society as a whole, such as smart phones, smart cities, smart homes, and smart cars, are emerging and being deployed. We are also enjoying an easier and more comfortable life brought about by smart devices. Survey results showed that 1) the innovation of self-driving technology for automobiles was the main reason for the commercialization of self-driving cars, and is a way to solve the traffic problem that is emerging around large city centers; 2) about 90 percent of traffic accidents are caused by the driver's cognitive, judgment, and manipulation mistakes; 3) the space is expected to expand to allow more activities within the means of transportation; and 4) core semiconductor technology enhancement is getting easier and more stabilized for self-driving technology deployment as the technology is improving significantly and moving toward the commercialization of self-driving cars globally[1].

In keeping with these self-driving innovation market and technology trends, we reemphasize the importance of core semiconductor technology development. So, the major purpose of this paper was to compare and analyze the competitive edge of major global semiconductor solution providers that are driving $\mathrm{SoC}$ platform strategy in the self-driving sector. We also examined 1) the status and flow of self-driving technology of traditional automobile manufacturers, 2) the entry strategy of global IT companies into the automobile and self-driving car market, and 3) the counterplan and milestones of Hyundai/Kia Motors and the Korean government to cope with the self-driving trend in prior and related studies. We finally looked at the competitiveness of self-driving solutions by major global semiconductor companies such as Nvidia, Intel and Qualcomm. Based on practical experience and advice from experts and professors who are engaged in the automobile industry and self-driving sector, we selected the verification and characteristics of competitive analysis for the three semiconductor companies by dividing them into five areas: 1) technology innovation, 2) knowledge management, 3) human resources, 4) sustainable management, and 5) commercial business.

\section{Related Studies}

\subsection{Meaning of Self-Driving}

The definition of self-driving means stable energy and stable driving by transporting designated people or objects at a designated time, such as conveyor belts that do not require human hands. Cars that can be fully autonomous are concentrated with state-of-the-art technologies such as semiconductors for AI, high-resolution maps, cameras, lasers, and voice-recognition AI. The entire self-driving system is very complex, and can be seen as a series of information 
processing processes called recognition, judgment, and manipulation through sensors and $\mathrm{AI}[2]$. Some of the latest vehicles to be released have what is called a self-driving support system, which meets the first and second stages of self-driving cars. They can be driven at a distance from the car in front of them, and can even slow down to the speed limit on the road. It is not uncommon to change lanes or overtake cars, but this provides the corresponding technology on some vehicles. From the third stage, a level of technology that reflects the will of the vehicle to a certain extent will be applied. Of course, drivers should be able to intervene immediately, but most of the vehicles drive on their own and use sensors, cameras, radar, and lidar to understand the situation to some extent. The fourth stage includes conditions under which limited judgment is made about driving. For example, people drive on their own in places like urban centers and highways, but may not be able to play their part in bumps or unexpected points. Of course, the driver will have to hold the steering wheel and drive. The fifth stage is literally the final evolutionary form. It means that a car judges all situations on its own and reflects them in its driving. The concept of a driver disappears and the person in the vehicle is just a passenger. It's the final destination and ultimate goal, but it's a difficult step to reach technically and commercially at this moment.

To develop self-driving cars, technologies in various fields such as hardware, software, artificial intelligence, and big data are combined. Hardware requires technical skills such as high-performance cameras, anti-collision devices and various functional sensors. Software requires the technology of a driving situation recognition and response system that comprehensively determines and controls the situation while driving. In particular, self-driving cars can be seen as a huge machine operated by software that is running efficiently through algorithms and programs.

\subsection{Status of Self-Driving Technology of Global Traditional Car Manufacturers}

GM and Ford, U. S. - based car companies, have been leading the pursuit of mass production business models based on the premise of production and procurement systems by vertical integration with gasoline car manufacturing where an internal combustion engine is the core. Although there were no reports as noticeable as Tesla's in the progress of commercialization of self-driving cars, GM in particular announced them for the driverless taxi ride sharing business along with the the fourth stage of self-driving cars and commercial launch goals by 2023[3]. Toyota in Japan has maintained its lead in automobiles around the world with a competitive advantage in production and operating systems. In particular, the company has established 
Assessment of Competitive Edge of Major Global Semiconductor Vendors for Self-Driving Solutions (Level 3 and Above)- Evaluation of Qualcomm, Intel, and Nvidia

domestic and foreign self-driving research institutes to implement rapid follow-up milestones through strategic technology partnerships, platform opening, and M\&As with start-up developers, as well as accumulating big players[4]. Germany was the birthplace of the 19th-century automobile industry. Daimler, Volkswagen, and BMW, Germany's Big Three, which have established solid status with a long history, are leading self-driving technology in the European region. Daimler, the leader in self-driving cars in particular, already had a driving device that keeps the car's speed constant in 1960. A Mercedes-Benz S-Class car equipped with an MCU completed a race from Munich, Germany, to Copenhagen, Denmark. In August 2013, an experimental vehicle drove autonomously on a general road between Mannheim and Portzheim in Germany. All recent models are equipped with a drive pilot and a partial self-driving system to assist in driving on highways. In addition, the company's "active lane switching assist," which automatically changes lanes when drivers manipulate turn signals, was the second fastest in the world after the Tesla S[5].

\subsection{Entry Strategy of Global IT Companies Into Automotive Industry}

Tesla and Google are leading the self-driving forces of Silicon Valley. Armed with IT and AI technology, these companies pursue an innovative business model that provides mobility services on the premise of fully self-driving cars and IOT device production experience that focuses on customer experience services. Navigant Research[6], a U.S.-based market research firm, released a report based on its assessment of self-driving technology companies. In the report, self-driving technology companies were largely divided into four groups: Leaders, Contenders, Challengers and Followers. The top group leader was Waymo, the self-driving car business division of Google's parent company Alphabet. In particular, Waymo received the highest score among the companies surveyed in terms of strategy and implementation. After Google launched its self-driving projects in 2009 and 2010, it began developing self-driving cars equipped with cameras, lidars and radars. It also announced an alliance called the Open Automotive Alliance (OAA) in 2014, which includes GM, Audi, Honda, Hyundai/Kia Motor and Nvidia. In December 2017, it established the subsidiary Waymo and started commercialization in earnest, supporting the world's highest three-stage self-driving and for the first time, introducing the concept of unmanned taxis that run when passengers call them and go to their destination. Tesla, another top leader selected by Navigant Research, has unique self-driving technology that is based on big data with more than 600,000 units of electric vehicles that use autopilot every day in all kinds of different situations across the United States. Global 
companies have reached almost perfect levels for self-driving in a normal driving environment, but accidents occur in unstructured and unexpected situations. In the case of vehicles equipped with autopilot functions such as Tesla Model S, it is equivalent to level 2 to level 3, which operates for $400 \mathrm{~km}$ on its own, but gives signals when users need to control it. It also introduced the concept of a car that evolves through software technology like a smart phone, which is always connected to the Internet. Features are added through software from day to day to improve driving performance without having to replace new hardware called vehicles. What do the two innovative companies such as Google and Tesla have in common are 1) their vision, in particular the CEO's philosophy and thought, 2) pursuit of thorough user experience, 3) building platforms and utilizing ecosystems in their business domains, and 4) efficient use of big data and AI.

\subsection{Self-Driving Counterplan of Hyundai/Kia Motors}

Hyundai/Kia Motor Group's first-generation ADAS integrated controller is powered based on an $\mathrm{MCU}$, but the second generation is a system that incorporates a number of accelerators dedicated to processing high-speed computing to handle the CPU, which is a higher-performance processor than MCU for enhanced cognitive (detection sensor), judgment (central control), and control (operator and actuator) functions. An additional vision processing unit (VPU) is applied. Hyundai/Kia Motor is in charge of developing the overall system, including algorithms for controllers, while Hyundai Mobis is in charge of developing hardware that converges sensors such as radars and lidars. In addition, Hyundai Autron's software platform would be included. Processors and solutions from Intel, Nvidia and TI are responsible for high-performance operations of self-driving of level 1 to level 2. During 2017 and 2018, seven controllers equipped with standard software platforms optimized for Hyundai/Kia Motor's specifications were applied to four models: the Genesis G70, Ioniq EV, the new Grandeur, and the Grandeur Hybrid. The Hyundai/Kia Motor Group, the world's top self-driving technology holding company, plans to develop software for self-driving cars that can be applied in common to the global auto industry by investing 2.4 trillion won, the largest investment in the Group's history, in 2019. They also have a vision to lead the ecosystem of self-driving cars beyond simple manufacturing, along with sales plans for key components. It has decided to set up a joint venture with Aptive. The Hyundai/Kia Motor Group's self-driving technology is still ranked 15th in the world, and it aims to secure level 4 technology by establishing the joint venture company Motional (formerly the Hyundai-Aptive 
Assessment of Competitive Edge of Major Global Semiconductor Vendors for Self-Driving Solutions (Level 3 and Above)- Evaluation of Qualcomm, Intel, and Nvidia

JV) and targets to start mass production of vehicles from 2024.

\subsection{Strategy of Korean Government}

According to forecasts by several market research institutes, self-driving cars are expected to grow rapidly between 2025 and 2035 after forming an initial market centered on North America and Europe, where there are few related regulations. In particular, according to a report by IHS (2019), global sales of self-driving cars will reach 600,000 units in 2025 and 21 million in 2035[7]. A report from PWC (2019) predicted the proportion of self-driving cars at level 3 or higher among new cars to be 6 percent in 2020, 25 percent in 2025, and 62 percent in 2030[8]. Klaus Schwab (2016) predicted in his book "The Fourth Industrial Revolution" that 10 percent of cars on U.S. roads by 2025 would be self-driving cars[9]. The automobile industry is Korea's leading flagship industry and has great front-to-back ripple effects, as the country's total manufacturing output accounted for 13.9 percent and value added was 57 trillion won. Since the Korean government is supporting technology development with the goal of commercializing level 3 self-driving cars in 2021, it seems necessary to create an ecosystem more quickly and effectively. In particular, the three core tasks of self-driving cars were decided to be safety, the development of evaluation technology, and the establishment of a test bed. It has also been pointed out that legal improvements for commercialization lacked because of the passive attitude of the government toward the commercialization of self-driving cars and national acceptance[10]. According to the results of the AV Readiness Index in 2018[11], the Netherlands was the most advanced country in technology development, with Singapore coming in second. Korea is in the top 10. The United States, Sweden and Germany, which have global car brands, ranked third, fourth, and sixth, respectively, while auto powerhouse Japan ranked only 11th. Among the top 10 were Britain (fifth), Canada (seventh), the United Arab Emirates (eighth), and New Zealand (ninth). Currently, Korean car makers have relatively inferior competitiveness in smart mobility technology and self-driving cars compared to major countries. The policy measures advocated for the Korean government's global cooperation are the promotion of a global data platform and the establishment of a joint R\&D center with Israel[12].

\section{Overview of Semiconductor Market and Three Companies}

\subsection{Automotive Semiconductor Market}


According to Gartner (2019) and WSTS (2019), the global semiconductor market is very large at \$47.6B (2018) and \$51B (2019), and along with the composition of non-memory (75\%) and memory $(25 \%)$, the markets for semiconductor manufacturers and sales companies rank in the order of telecommunications (32.4\%), computers (29.6\%), general consumer/industrial (23.4\%), automobiles $(11.5 \%)$, and other (3.4\%). Qualcomm stands out in telecommunications, while Intel stands out in the computing industry. Data from Gartner (2019) and WSTS (2019) also show that the automotive semiconductor market is expected to grow by more than $10 \%$ every year, with an average semiconductor value of $\$ 700$ per car in 2025 and a $\$ 63 \mathrm{~B}$ market size[13][14]. It is expected that the company will enter the high value-added automotive semiconductor market and compete fiercely with the existing leader NXP and Infineon in connected, electric vehicles, and self-driving. Korea-based Samsung Electronics Co. and SK Hynix Inc. and U.S.-based Micron Memory Inc. are currently leading the global memory market, and demand for memory capacity is also on the rise in the auto market.

\subsection{Company Overviews and Self-Driving Technology History of Three Semiconductor Companies}

There is fierce competition among global semiconductor companies to take the lead in the semiconductor market in the field of self-driving cars, and Nvidia has established itself as a partner of global auto makers with the most advanced competitiveness in self-driving systems early on, while Intel and Qualcomm are fiercely chasing each other. We give overall company profiles and self-driving technology histories of these three companies in detail in this section prior to competitive edge analysis.

\subsubsection{Nvidia}

Nvidia has headquarters in Silicon Valley along with about 12,000 employees globally and 9.7 billion dollars in sales (the world's eighth-largest semiconductor sales), Jenson Huang is the CEO of Nvidia, founded in 1998 and listed on the U.S. Nasdaq exchange. Recently it passed Intel's market cap, taking a 70\% share of the global GPU market and leading in AI technology. Huang made an excellent decision to decrease the mobile business and change business portfolios such as games (51\%), data center (27\%), commercial design (11\%), and automobiles $(6 \%)$. It No. 1 in patent rights in the AI semiconductor field globally (Source: company website of Nvidia). 
Assessment of Competitive Edge of Major Global Semiconductor Vendors for Self-Driving Solutions (Level 3 and Above)- Evaluation of Qualcomm, Intel, and Nvidia

\subsubsection{Intel}

Intel has headquarters in Santa Clara, USA along with about 45,000 employees globally and $\$ 65.8$ billion in sales as the world's No. 1 semiconductor provider in 2019. Currently CEO Robert Swan, who was previously the CFO of Intel, is leading the company and acquired the self-driving solution company Mobileye (Israel-based, \$15.3B) in March 2017. It relocated outstanding engineering resources from the existing computing field to automobiles to reinforce self-driving workforce and diversify its business portfolio beyond the mobile, server, and computing businesses (Source: company website of Intel).

\subsubsection{Qualcomm}

Qualcomm has headquarters in San Diego, U.S. along with about 40,000 employees globally and $\$ 28.3$ billion in sales and was No. 6 in the world in semiconductor sales in 2019. Steve Molenkopf has been CEO since 2013. Qualcomm is leading the global mobile industry as total solution provider. Molenkopf has made many efforts to diversify its business portfolio to computing, IOT, RF, and automotive to leverage its excellent and proven wireless platforms in $\mathrm{H} / \mathrm{W}, \mathrm{S} / \mathrm{W}$, and $5 \mathrm{G}$ technology and invests $20 \%$ of total sales in R\&D every year. Qualcomm merged with CSR (UK, \$3.5B), an automotive connectivity semiconductor provider, in 2013 (Source: company website of Qualcomm).

\section{An Analysis of the Competitiveness of Self-Driving Technology of Three Semiconductor Companies}

As previously reviewed, we extracted major factors for a competitive edge study using appropriate feedback and validated data provided by experts and professors who are engaged in both self-driving solutions and the automobile industry as this research's design and methodology. The analysis of competitiveness of the three semiconductor companies was divided into five areas: 1) technology innovation, 2) knowledge management, 3) human resources, 4) sustainable management, and 5) commercial business, in consultation with experts who carried out precedence case studies with semiconductor companies with respect to competitive edge. The companies were ranked first, second, and third, comparatively, for each area, and were marked H (excellent level, first), M (normal level, second) and L (inferior level, third), respectively. Overall ranking and competitiveness are mentioned in each conclusion section. We summarized the overall evaluation results of comparison made among the three 
companies in the conclusions and implications part of this paper.

\subsection{Technology Innovation}

Traditionally, the main types of technological innovation were the invention or development of new products, the introduction of new production methods, the invention of new technologies, the pioneering of new markets, the discovery and use of new materials or parts, and the creation of new organizations to increase productivity. However, in the global low-growth trend and fierce competition, entrepreneurs should consider creative destruction by breaking old products, production methods, and distribution structures with technology innovation[15]. The participants discussed the future of mobility and implications for climate change, clean and livable cities, the traditional utility power grid, and the changing regulatory landscape. They also reemphasized the importance of SoC development for self-driving cars[16]. The cars of the future are evolving into mobile living spaces where eco-friendly self-driving technology is possible. The role of electrically processing, controlling, and ordering information in the vehicle is essential, and the auto semiconductor is expected to play a key role in expediting the commercialization of self-driving technology even to level 4 and above in 2025 and beyond[17][18]. In this paper, the technology innovation sector was limited to self-driving technology. The competitiveness of the three semiconductor companies' product performance (CPU) and future roadmap, software scalability and HW platform strategy, design capability, quality control, and manufacturing process were mainly examined. Nvidia has secured major client companies including Baidu, Daimler, Ford, Toyota, Volkswagen, Volvo, and Voyage in its leading position in self-driving technology. It was superior in product performance, quality, and manufacturing, and on the other hand, it showed lower expectations in SW expandability. Intel is working closely with Hyundai/Kia Motors as a self-driving technology partner of level 2 and level 3 with the acquisition of Mobileye. Although there is a competitive edge in SW and algorithm with Mobileye, it has not shown a comparative advantage in HW platform strategy or manufacturing/quality sector. Qualcomm is a latecomer, but it has been selected as an ADAS and autonomous driving partner with GM and BMW in Europe and is working closely with them with the aim of commercializing it in 2023. Although its experience with self-driving technology is short, it showed a comparative advantage in HW platform, SW scalability, and new product release. 
Assessment of Competitive Edge of Major Global Semiconductor Vendors for Self-Driving Solutions (Level 3 and Above)- Evaluation of Qualcomm, Intel, and Nvidia

[Table 1] Comparison of Competitiveness in Technology Innovation

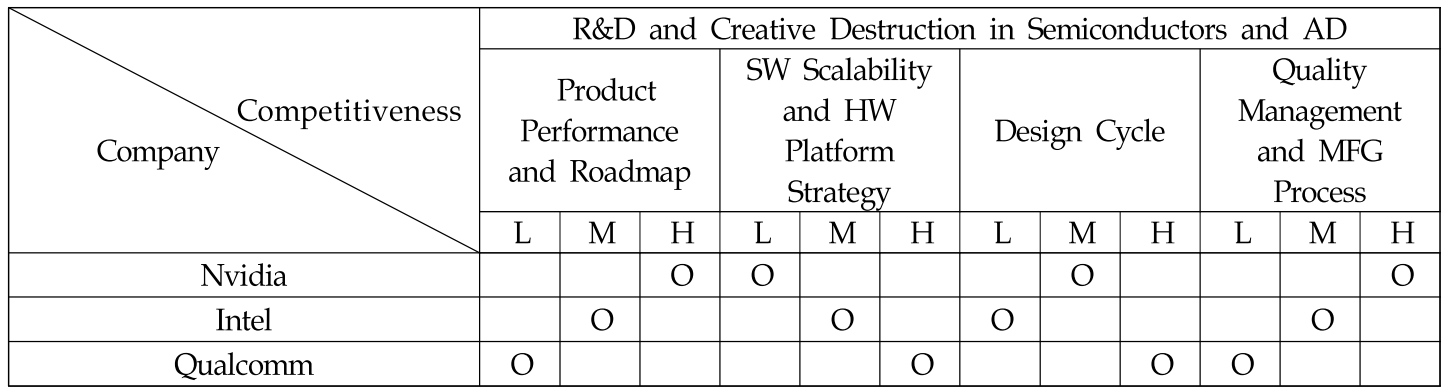

Data: Analysis and theorem after in-depth interview in August 2020 (Low : inferior, Medium : normal, High : excellent)

Conclusion 1. Nvidia's overall excellent competitiveness in performance and quality, while Intel and Qualcomm are currently ranked second in technology innovation. Qualcomm in particular showed strengths in SW scalability and HW platform.

\subsection{Knowledge Management}

Recently, global innovators have also been making great efforts to accelerate knowledge management to build a systematic foundation for intangible assets such as market assets, human assets, intellectual property assets, infrastructure assets, etc. So C-based self-driving technology is not only to enhance the quality of service in the automotive industry, but also to promote the value of the automotive company's business[19]. In this paper, patent rights, organizational innovation, employee innovation mind set, and customer satisfaction items were set around the automobiles of the three semiconductor companies as knowledge management sectors. Nvidia was found to have a comparative advantage in terms of IPR, organizational innovation and employee mind set in the knowledge management sector as a whole. What's important is that CEO Jenson Huang is not tied to short-term performance by emphasizing ideas and innovations to employees and presenting the company's management goals from a mid-/long-term perspective. Intel continues to promote IPR and organizational innovation with the acquisition of Mobileye, an Israeli venture company, but the synergy effect of knowledge management is halved due to difficulties in communication and conflicts between organizations resulting from different corporate cultures. Qualcomm has established its patent management and innovation spirit in the mobile business as important policies of the company, but it has been analyzed that more time and investment are needed to exert the effect of knowledge management in the automotive business, while it showed a comparative advantage in the area of customer satisfaction as a latecomer. 
[Table 2] Comparison of Competitiveness in Knowledge Management

\begin{tabular}{|c|c|c|c|c|c|c|c|c|c|c|c|c|}
\hline \multirow{3}{*}{ Company } & \multicolumn{12}{|c|}{ Biz/Org. Portfolio Flexibility and Automotive Innovation Mindset } \\
\hline & \multicolumn{3}{|c|}{ IPR } & \multicolumn{3}{|c|}{$\begin{array}{l}\text { Organization } \\
\text { Innovation }\end{array}$} & \multicolumn{3}{|c|}{$\begin{array}{c}\text { Employee } \\
\text { Mindset }\end{array}$} & \multicolumn{3}{|c|}{$\begin{array}{r}\text { Customer } \\
\text { Satisfaction }\end{array}$} \\
\hline & $\mathrm{L}$ & $\mathrm{M}$ & $\mathrm{H}$ & $\mathrm{L}$ & $\mathrm{M}$ & $\mathrm{H}$ & $\mathrm{L}$ & $\mathrm{M}$ & $\mathrm{H}$ & $\mathrm{L}$ & $\mathrm{M}$ & $\mathrm{H}$ \\
\hline Nvidia & & & $\mathrm{O}$ & & & $\mathrm{O}$ & & & $\mathrm{O}$ & $\mathrm{O}$ & & \\
\hline Intel & & $\mathrm{O}$ & & & $\mathrm{O}$ & & $\mathrm{O}$ & & & & $\mathrm{O}$ & \\
\hline Qualcomm & $\mathrm{O}$ & & & $\mathrm{O}$ & & & & $\mathrm{O}$ & & & & $\mathrm{O}$ \\
\hline
\end{tabular}

Data: Analysis and theorem after in-depth interview in August 2020 (Low : inferior, Medium : normal, High : excellent)

Conclusion 2. The three companies' promotion of knowledge management was closely linked to the unique corporate culture and vision of the companies, making it relatively difficult to compare and evaluate them. The Nvidia CEO's strong drive and leadership in innovation are believed to be the driving force behind the company's transformation and maintaining its lead in self-driving technology as well as remarkable rise in the new growth sector.

\subsection{Human Resources}

In terms of human resources development (HRD), most global companies have recently introduced a strategic human resource development system to implement a tool and method aligned with the goals of the organization to increase the quality and productivity of individual human resources and the ultimate competitive edge of the companies. Employees can also make progress in terms of both capacity development and career path management in the future. Training of successors, securing technical personnel, and re-education of employees play significant roles in corporate competitiveness. That is the reason why HRD is becoming more and more critical in the semiconductor industry. In particular, engineering resources are the most important asset to take product leadership in semiconductor companies to lead the companies to global growth[20]. In this paper, not only the CEO leadership of the three semiconductor companies, but also the ability of the management or team leaders in self-driving technology were taken as indicators along with R\&D resources capability, retraining tools for employees, and compensation level for performance. Nvidia and Intel have good human resources for $R \& D$ in self-driving technology. Qualcomm is reinforcing $R \& D$ as a latecomer with the continuous selection of competent resources in the automotive business with strong support from its $\mathrm{CEO}$ or by transfer and re-education in the internal mobile $R \& D$ sector, but it still shows a difference or is inferior in securing human resources compared with Nvidia and Intel's self-driving R\&D capability. 
Assessment of Competitive Edge of Major Global Semiconductor Vendors for Self-Driving Solutions (Level 3 and Above)- Evaluation of Qualcomm, Intel, and Nvidia

[Table 3] Comparison of Competitiveness in Human Resource

\begin{tabular}{|c|c|c|c|c|c|c|c|c|c|c|c|c|}
\hline \multirow{3}{*}{ Company } & \multicolumn{12}{|c|}{ Company Strategy/Execution for Human Resource Development } \\
\hline & \multicolumn{3}{|c|}{$\begin{array}{c}\text { CXO's } \\
\text { Leadership }\end{array}$} & \multicolumn{3}{|c|}{ R\&D Resource } & \multicolumn{3}{|c|}{$\begin{array}{l}\text { Employee } \\
\text { Training }\end{array}$} & \multicolumn{3}{|c|}{ Compensation } \\
\hline & $\mathrm{L}$ & $\mathrm{M}$ & $\mathrm{H}$ & $\mathrm{L}$ & $\mathrm{M}$ & $\mathrm{H}$ & $\mathrm{L}$ & $\mathrm{M}$ & $\mathrm{H}$ & $\mathrm{L}$ & $\mathrm{M}$ & $\mathrm{H}$ \\
\hline Nvidia & & $\mathrm{O}$ & & & $\mathrm{O}$ & & & $\mathrm{O}$ & & & & $\mathrm{O}$ \\
\hline Intel & $\mathrm{O}$ & & & & & $\mathrm{O}$ & $\mathrm{O}$ & & & $\mathrm{O}$ & & \\
\hline Qualcomm & & & $\mathrm{O}$ & $\mathrm{O}$ & & & & & $\mathrm{O}$ & & $\mathrm{O}$ & \\
\hline
\end{tabular}

Data: Analysis and theorem after in-depth interview in August 2020 (Low : inferior, Medium : normal, High : excellent)

Conclusion 3. Nvidia is providing the benefit of stock compensation to employees to continuously secure competent resources. Intel has secured excellent technical personnel with the acquisition of Mobileye, and that company's previous team is currently leading SW and algorithm on self-driving technology, while Qualcomm, along with strong support from its $\mathrm{CEO}$, is implementing a strategy to redeploy and retrain their excellent mobile $R \& D$ workforces.

\subsection{Sustainable Management}

In the 2000s, the sustainable management paradigm emerged as the demand for corporate social responsibility increased sharply because economic growth was achieved to some extent and interest in the environment and social problems spread throughout society. Therefore, the trend of sustainable management prior research is more emphasized in economic and financial areas. For sustainable management, it is needed to develop a unified theoretical perspective to understand business model innovation that definitely improves the economic, environmental, and social performance of the organization, in particular readdressing 1) business model innovation, 2) sustainable management innovation, 3) network theory, 4) stakeholder theory, and 5) product-service system[21]. In this paper, we mainly looked at the companies' economic reliability and stability, transparency indicators, social reputation and accountability, cooperation chart and eco-friendly contributions, and ethical management policy and fair trade will. Nvidia is a leading player in self-driving technology and has definitely stood out in economic indicators. It is leading other rivals in sales growth, profit taking, and order backlogs, while Intel is providing a lot of support for expanding its ecosystem with global innovators with Mobileye. In addition, the company is paying special attention to its headquarters in this area as it often files unfair trade suits against governments around the world, like Qualcomm. It estimates that as a latecomer, sales related to self-driving technology will take place after 2023 
or 2024, while it emphasizes the morality and ethics of employees around the world as a higher priority at the headquarters level related to the sustainable management sector.

[Table 4] Comparison of Competitiveness in Sustainable Management

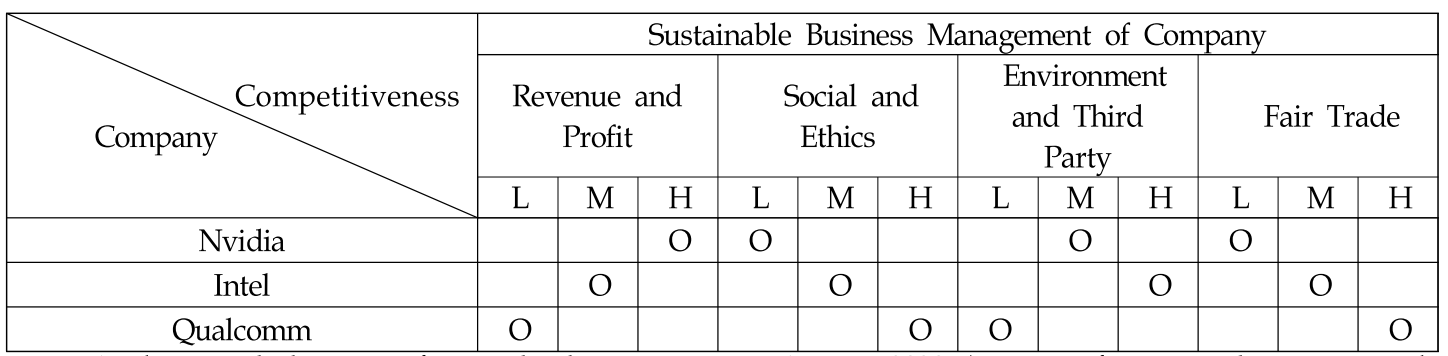

Data: Analysis and theorem after in-depth interview in August 2020 (Low : inferior, Medium : normal, High : excellent)

Conclusion 4. Nvidia has overall excellent position in financial and economic indicators, while Intel is leading the realization of sustainable management in the area of business environment in close cooperation with its surrounding ecosystem partners. Qualcomm has been found to be leading the social and ethic sector with the realization of fair trade and re-education on moral cultivation for employees globally.

\subsection{Commercial Business}

The traditional concept of comparing product quality, service capability, and revenue growth was the basis for corporate competitiveness, while the accumulated sums of strategic partnerships, technical support capabilities, and supply chain assistance level for customers have become increasingly important under a low growth trend and severe competition environment globally since the 2000s. In this paper, we closely focused only on evaluating price competitiveness, service and procurement capability, technical support, and loyalty to the customer in the self-driving sector. In particular, each item in the business sector has become an important criterion for adopting solutions and securing competitiveness from customers in self-driving technology. Recently, customers directly communicate with the headquarters by explaining their strategies, policies, intimacy, and customer support requests. Nvidia proved its excellence in direct technical support from the head office and Intel was analyzed as having a comparative advantage in terms of price competitiveness. Qualcomm, which is a latecomer, was normal in technical support and price competitiveness, but it has a relatively higher competitive edge in contract construction, sample support, and communication along with 
Assessment of Competitive Edge of Major Global Semiconductor Vendors for Self-Driving Solutions (Level 3 and Above)- Evaluation of Qualcomm, Intel, and Nvidia

customers by getting active support from its headquarters from the early stages of a project, which is characterized by growing business competitiveness in the self-driving sector in the end.

[Table 5] Comparison of Competitiveness in Commercial Business

\begin{tabular}{|c|c|c|c|c|c|c|c|c|c|c|c|c|c|}
\hline \multirow{2}{*}{$\begin{array}{c}\text { Competitiveness } \\
\text { Company }\end{array}$} & \multicolumn{10}{c|}{ erms and Conditions on Semiconductor Business for Semiconductor } \\
and AD
\end{tabular}

Data: Analysis and theorem after in-depth interview in August 2020 (Low : inferior, Medium : normal, High : excellent)

Conclusion 5. Qualcomm is analyzed as excellent in feedback on response and logistic assistance to meet customers' needs on time. Intel and Nvidia each showed price competitiveness in the commercial area and a comparative advantage in headquarters' technical support.

\section{Conclusions and Implications}

This study focused on comparing and analyzing the competitiveness of self-driving solutions of the three major semiconductor companies in the automobile market. In summary, Nvidia held an overall No. 1 position in technology innovation, knowledge management, human resources, and sustainable business while taking leadership in self-driving technology. On the other hand, Intel has comparative strengths in human resources (engineering resources) and sustainable business (ecosystem partner development) by making a synergy effect with Mobileye. Qualcomm in particular held a better position in sustainable business (ethics and fair trade) and commercial business (supply chain and communication) as a fast follower.

Based on a study of previously published papers and related work and the comprehensive evaluation results of the competitiveness of the three global semiconductor companies in self-driving technology in this paper, we would like to present practical and policy implications for the future enhancement and development of the Korean automobile industry and recall key messages for the spread of self-driving technology and the development of domestic semiconductor companies in Korea. First, in order to spread self-driving technology and secure 
competitiveness, the Korean government should set up a prior national project to inject a budget and implement a top strategy for fostering industrial and academic technical personnel. Second, Hyundai/Kia Motors Group should focus on expanding its M\&As and global partnerships to tap into the global market with its CEO's strong innovation execution strategy. In addition, self-driving technology should be adopted efficiently to create a competitive advantage over global car makers in terms of commercial competitiveness. Third, the automotive semiconductor market is a high growth and high value industry with more than 10 percent annual growth. Local semiconductor manufacturers including Samsung and SK Hynix will also have to try to expand the development of both non-memory and memory products related to automobiles and ultimately diversify their businesses portfolios. Fourth, local semiconductor solution development execution and competitiveness increase related to self-driving technology have been reemphasized. Also, surrounding industries such as platforms, software, contents, and security sectors must be revived to expect the continued growth of domestic cars.

In this study, the competitiveness of the three global semiconductor companies was compared and analyzed as the main topic. Practical and policy proposals were also made for the development and optimization of self-driving solutions for the Korea government as well as local industry and domestic companies. However, in this paper, it is believed that there will be certain limitations to draw conclusions related to self-driving solutions by conducting interviews with experts and professors and analyzing cases of the three major global semiconductors companies. It is also regrettable that the we could not carry out a quantitative analysis study based on a survey with questionnaires, hypothesis verification, and an empirical process. In future studies, we would like to expand the size of the case analysis and draw conclusions using statistical analysis.

\section{References}

[1] Report of KATECH, Development Trend and Critical Issues of Autonomous Mobility, KATECH, (2020)

[2] Young-Seok Park, Ji-Hyun Yang, Se-Joon Lim, Development of Complexity Index and Predictions of Accident Risks for Mixed Autonomous Driving Levels, IEEE International Conference on Systems, Main, and Cybernetics (SMC), (2018), October 7-10; Miyazaki, Japan.

[3] Jae-Hoon Hyun, The Strategy of GM for the Development of Autonomous Driving Technology and Related Policies, Journal of the Korea Academia-Industrial cooperation Society, (2020), Vol.21, No.3, pp.51-56.

[4] Toyota Strategic Report, Toyota Research, Japan: Toyota, (2019) 
[5] Mckinsey, Mckinsey Annual Report, USA: McKinsey \& Company, (2019)

[6] Naviant, Naviant Annual Report, USA: Naviant Research, (2019)

[7] The IHS Autonomous Driving Materials Report, IHS, (2019)

[8] 4th ICO/STO Report, March Edition, PWC, (2019)

[9] Klaus Schwab, The Fourth Industrial Revolution, Korea: Sae-Ro-Un-Hyun-Jae, (2016)

[10] Jae-Sam Lee, A Study of Act Improve of Commercialization of Autonomous Vehicle, Hannam Journal of Law \& Technology, (2019), Vol.25, No.4, pp.117-160.

[11] 2018 Autonomous Vehicles Readiness Index, KPMG, (2018)

[12] Hee-Tae Yang, Policy instruments for international cooperation to improve smart mobility competitiveness: focusing on South Korea \& Israel, The Journal of Korean Institute of Communications and Information Sciences, (2018), Vol.44, No.5, pp.992-999.

[13] Gartner Annual Report, Gartner, (2019)

[14] WSTS Annual Report, World Semiconductor Trade Statistics, (2019)

[15] Jae-Kyung Kim, Absolute Advantage + ONE Strategy, Hivison, (2020)

[16] Paul R. Donnellan, The Future of Mobility-Electric, Autonomous, and Shared Vehicles, IEEE Engineering Management Review, (2019), Vol.46, No.4, pp.16-18.

[17] Dae-Kyung Nam, Kyung-Hyun Choi, Technology Trend Analysis in the Automotive Semiconductor Industry using Topic Model and Patent Analysis, Journal of KOTIS (Korea Technology Innovation Society), (2018), Vol.21, No.3, pp.1155-1178.

[18] Thomas Schiller, Nikolaus Helbig, Matthias Nagl, How can European automakers thrive in the new mobility ecosystem?, The automotive value chain in 2025 and beyond, Part of a deloitte series on the future of mobility, USA: Deloitte Insights, (2020)

[19] Yeong-Min Kwon, Hyung-Joo Kim, Kyung-Il Im, Jae-Hwan Kim, Woong-Bee Son, A Research on Forecasting Change and Service Direction for the Future Mobility System, The Journal of The Korea Institute of Intelligent Transport Systems, (2020), Vol.19, No.3, pp.100-115.

[20] Young-Hwa Kwon, A Case Study on the Global Growth of Semiconductor Companies by Human Resource Management, Development and Strategy, Journal of Korea Research Association of International Commerce, (2016), Vol.16, No.4, pp.33-67.

[21] Steve Evans, Doroteya Viadimirova, Maria Holgado, Kirsten Van Fossen, Miying Yang, Elisabete A. Silva, Claire Y. Barlow, Business Model Innovation for Sustainability: Toward a Unified Perspective for Creation of Sustainable Business Models, Biospectroscopy, (2017), Vol.26, No.5, pp.597-608. 